\title{
Influência do hábitat na estrutura da comunidade de macroinvertebrados aquáticos associados às raízes de Eichhornia crassipes na região do Lago Catalão, Amazonas, Brasil
}

\begin{abstract}
Aline LOPES ${ }^{1}$, Joana D’Arc de PAULA², Silvia Fernanda MARDEGAN ${ }^{3}$, Neusa HAMADA ${ }^{4}$, Maria Teresa Fernandez PIEDADE 5

\section{RESUMO}

Por possuírem ciclos de vida curtos, os macroinvertebrados aquáticos podem responder rapidamente às modificações ambientais, alterando a estrutura das suas populações e comunidades. O objetivo deste estudo foi determinar se há relação entre a composiçáo de macroinvertebrados aquáticos associados a bancos de Eichhornia crassipes o gradiente de condutividade elétrica da água e a biomassa das raízes destes bancos. No pico da cheia de 2005, 21 bancos de macrófitas aquáticas flutuantes dominados por $E$. crassipes foram amostrados no rio Negro (baixa condutividade $\leq 30 \mu \mathrm{S} \mathrm{cm}^{-1}$ ), na confluência entre os rios Negro e Solimóes (média condutividade $>30 \mu \mathrm{Sm}^{-1} \mathrm{e} \leq 50 \mu \mathrm{S} \mathrm{cm}^{-1}$ ), e no rio Solimóes (alta condutividade $>50 \mu \mathrm{Sm}^{-1}$ ). Foram encontrados 1707 macroinvertebrados aquáticos, distribuídos em 14 ordens e 35 famílias. A abundância de invertebrados aquáticos foi maior em bancos na confluência das águas. $\mathrm{O}$ aumento da biomassa das raízes de $E$. crassipes levou a um aumento da abundância e da riqueza de famílias de macroinvertebrados. A abundância dos coletores-catadores, coletores-filtradores e raspadores variou com o tipo de água, e apenas a abundância dos coletores-catadores e coletores-filtradores variou em função da biomassa das raízes. A riqueza de famílias dos raspadores variou em função do tipo de água. A organização da comunidade depende do gradiente de biomassa de raízes, indicando a importância da estrutura do hábitat para o estabelecimento dos macroinvertebrados.
\end{abstract}

PALAVRaS-Chave: Amazônia Central, rio Negro, rio Solimóes.

\section{Influence of habitat on community structure of macroinvertebrate associated with roots of Eichhornia crassipes in the Lake Catalão, Amazonas, Brazil}

\begin{abstract}
Owing to their short life cycles aquatic macroinvertebrates may quickly respond to environmental modifications by changing the structure of their populations and communities. The aim of this study was to determine the relationship between the composition of aquatic macroinvertebrates associated with Eichhornia crassipes stands, the gradient of water conductivity and biomass of the roots of these stands. At the peak of the floods of 2005, 21 banks of floating aquatic macrophytes dominated by $E$. crassipes were sampled in the Solimóes River (high conductivity $>50 \mu \mathrm{S} \mathrm{cm}^{-1}$ ), the confluence of these the rivers Solimóes and Negro (medium conductivity $>30 \mu \mathrm{S} \mathrm{cm}^{-1}$ and $\leq 50 \mu \mathrm{S} \mathrm{cm}^{-1}$ ) and Negro River (low conductivity $\leq 30 \mu \mathrm{S} \mathrm{cm} \mathrm{cm}^{-1}$ ). A total of 1707 aquatic macroinvertebrates were found, divided into 14 orders and 35 families. The abundance of aquatic invertebrates was higher in stands in the rivers confluence. The increased biomass of roots of E. crassipes led to an increase of abundance and richness of families of macroinvertebrates. The abundance of collector-gatherers, filter feeders and collector-scrapers varied with the type of water; only the abundance of collector-gatherers and collector-filter-varied with the root biomass. The abundance of collector-gatherers, filter feeders and collector-scrapers varied with the type of water, and only the richness of collectors and filter feeders varied with the root biomass. The family richness of collector-scrapers varied with the type of water. The organization of the community depends on the gradient of root biomass, showing that habitat structure is an important factor for the establishment of macroinvertebrates.

KEYWORDS: Central Amazonian, Negro River, Amazon River.

\footnotetext{
1 Programa de Pós-Graduação em Ecologia Instituto Nacional de Pesquisas da Amazônia - INPA CP 47869011 -970 Manaus - AM, Brasil. alopesmga@gmail.com

2 Programa de Pós-Graduação em Ecologia Instituto Nacional de Pesquisas da Amazônia - INPA CP $47869011-970$ Manaus - AM, Brasil. jddpaula@gmail.com

3 Programa de Pós-Graduação em Ecologia. Instituto Nacional de Pesquisas da Amazônia. silmardegan@gmail.com

${ }^{4}$ Coordenação de Pesquisas em Entomologia. Instituto Nacional de Pesquisas da Amazônia. - INPA CP 478 69011-970 Manaus - AM, Brasil. nhamada@inpa.gov.br

${ }^{5}$ Coordenação de Pesquisas em Biologia Aquática. Instituto Nacional de Pesquisas da Amazônia. - INPA CP 478 69011-970 Manaus - AM, Brasil. maitepp@inpa.gov.br

* Endereço atual: Programa de Pós-graduação em Ecologia Aplicada. Universidade de São Paulo. Av. Centenário 303 São Dimas $13416-000$ - Piracicaba, SP - Brasil
} 


\section{INTRODUÇÃO}

$\mathrm{Na}$ Amazônia, a vegetação das áreas alagáveis é estruturada pelo pulso de inundação, havendo uma marcada sazonalidade de crescimento e decomposição das macrófitas aquáticas (Junk et al. 1989; Junk e Piedade 1997). As drásticas alteraçôes ambientais decorrentes da flutuaçáo média de 10 metros no nível da água, entre o pico das cheias e o pico das águas baixas dos grandes rios da Amazônia Central, definem uma fase aquática e uma fase terrestre ao longo do ano (Junk et al. 1989) que provocam elevadas perdas sazonais para a maioria dos organismos (Junk e Piedade 1997). Essa sazonalidade ocasiona a mortalidade de artrópodes (Adis e Junk 2002; Franklin et al. 1997; Martius 1987), plantas lenhosas (Larcher 2000; Taiz e Zeiger 2004) e herbáceas (Piedade et al. 2010a). As perdas decorrentes dessa pressão ambiental tendem a ser recuperadas por meio de estratégias adaptativas como crescimento rápido, maturidade precoce, altas taxas reprodutivas e outras adaptaçôes morfológicas, fenológicas e comportamentais.

A taxa de crescimento de macrófitas aquáticas flutuantes é diretamente proporcional à intensidade e quantidade de luz e nutrientes disponíveis (Petrucio e Esteves 2000). Na bacia amazônica, a distribuição dos nutrientes ocorre de acordo com a origem dos rios. O rio Negro, de águas pretas, tem suas nascentes no antigo escudo pré-cambriano das Guianas ou nos sedimentos terciários da bacia Amazônica, possuindo uma baixa carga de sedimentos e baixo $\mathrm{pH}$, sendo considerado pobre em nutrientes (Sioli 1968; Junk 1983). Durante um ciclo hidrológico, a condutividade elétrica das suas águas pode variar de $8,6 \mu \mathrm{cm}^{-1}$ na seca a $13,8 \mu \mathrm{S} \mathrm{cm} \mathrm{cm}^{-1}$ na enchente, e o pH varia de 4,35 na enchente a 5,8 na seca (Sousa 2008). $\mathrm{O}$ rio Solimões, de águas brancas, nasce na região andina $\mathrm{e}$ pré-andina, possuindo alta carga de sedimentos e $\mathrm{pH}$ quase neutro, sendo considerado de elevada produtividade natural (Sioli 1968; Junk 1983). A condutividade elétrica do rio Solimóes ao longo de um ciclo hidrológico pode variar de 76,8 $\mu \mathrm{S} \mathrm{cm}{ }^{-1}$ na cheia a $112,4 \mu \mathrm{Sm}^{-1}$ na vazante, e o $\mathrm{pH}$ de 6,15 a 7,29 na vazante (Sousa 2008). Apesar da concentração de nutrientes variar sazonalmente nos rios Solimões e Negro, a carga de elementos entre os dois sistemas sempre é maior no rio Solimôes que no Negro (Sousa 2008). A baixa qualidade nutricional aliada ao baixo $\mathrm{pH}$ do rio Negro são os principais fatores que limitam o desenvolvimento de macrófitas aquáticas nessa área (Junk e Mello 1990) ao contrário do rio Solimões onde a abundância de macrófitas aquáticas é alta (Junk e Piedade 1993).

As espécies de macrófitas aquáticas, por possuírem uma complexidade estrutural de folhas e raízes espécie-específica, abrigam diferentes diversidades de invertebrados aquáticos (Cronin et al. 2006). A macrófita aquática flutuante Eichhornia crassipes (Mart.) Solms se destaca devido à densa massa de raízes, que são hábitats para peixes, alevinos, insetos e outros organismos aquáticos (Junk e Howard-Williams 1984). Eichhornia crassipes é uma das espécies mais abundantes na regiấo amazônica (Junk e Piedade 1997) e sua distribuição se estende a todas as regióes tropicais do planeta (Sculthorpe 1985).

As macrófitas aquáticas aumentam a complexidade do hábitat e servem como alimento ou abrigo para muitos organismos que habitam as zonas aquáticas como: Diptera, Trichoptera, Ephemeroptera, Odonata (Junk 1973; Cheruvelil et al. 2000; Cronin et al. 2006; Ali et al. 2007). Alguns macroinvertebrados aquáticos são importantes decompositores de detritos, sendo amplamente utilizados como bioindicadores de qualidade de água, devido a seu hábito sedentário e ciclos de vida curtos que permitem que eles reflitam rapidamente as modificaçóes ambientais na estrutura das suas populaçôes e comunidades (Resh et al. 1996).

A alta diversidade biológica dos macroinvertebrados aquáticos propicia uma maior variabilidade de respostas frente a diferentes tipos de impactos ambientais (Resh et al. 1996). Esses organismos têm uma ampla distribuição nos ambientes aquáticos onde colonizam a própria coluna de água, ou habitam os sedimentos, as raízes e as partes submersas de macrófitas aquáticas (Merritt e Cummnis 1996). As características físicoquímicas do ambiente aquático e a biomassa, composição das espécies e duração sazonal da vegetação aquática podem determinar a composiçáo e abundância de macroinvertebrados em um dado ambiente (Van den Berg et al. 1997), o que foi confirmado para a região amazônica por Nessimian et al. (1998) e Callisto et al. (1998, 2001).

Enquanto a distribuição de organismos aquáticos em ecossistemas delimitados como lagos pemite inferir sobre os processos que ocorrem em uma escala abrangente em nível ecossistêmico, os gradientes ambientais, como variaçóes de condutividade, salinidade, turbidez, entre outros, permitem analisar a influência de fatores locais sobre a comunidade (Bohonak e Jenkins 2003). Esses gradientes podem ser facilmente observados nas regioes de confluencia de rios, onde as comunidades estáo sujeitas a uma repentina substituicao de especies devido ao gradiente ambiental imposto (Holland 1988).

A conectividade de ambientes na mesma planície de inundação propicia a migração de organismos para os locais com condiçôes físicas e químicas mais adequadas as suas exigências (Amoros e Bornette 2002). Essa situação de conectividade é encontrada nas regiôes de confluência dos grandes rios amazônicos permitindo que, com o favorecimento dos ventos e correntes, macrófitas flutuantes como E. crassipes, abundantes nas férteis várzeas, possam chegar às águas mistas e pretas adjacentes, mesmo que esses náo sejam os ambientes ideais para seu crescimento. 
O estabelecimento de diversos organismos é limitado pela qualidade nutricional da água, e os gradientes naturais estabelecidos nesses locais são laboratórios apropiados para testar hipóteses. Partindo do pressuposto que as disponibilidades de hábitat e nutrientes são importantes para a estruturação da comunidade de invertebrados aquáticos, o objetivo deste estudo foi testar se a comunidade de macroinvertebrados que ocorre nos distintos tipos de água é estruturada pela qualidade nutricional do sistema, avaliada por parâmetros físico-químicos da água, e/ou pela disponibilidade de hábitat, avaliada pela biomassa de raízes de E. crassipes.

\section{MATERIAL E MÉTODOS}

O estudo foi realizado no lago Cataláo (3 09' 47"S e $\left.059^{\circ} 54^{\prime} 29^{\prime \prime} \mathrm{W}\right)$, área alagável, no encontro dos rios Negro e Solimôes, próxima à cidade de Manaus, Amazonas, Brasil (Figura 1), no mês de junho de 2005. Esse período corresponde à fase de cheia, quando o lago está conectado ao rio Solimóes por um curto canal (Leite et al. 2006). Esta conexão possibilita a migração de macrófitas aquáticas do lago para o rio Negro. Estas plantas são dispersadas pela força da correnteza e levam nas raízes diversas espécies da fauna aquática. $\mathrm{Na}$ fase terrestre esse lago fica quase completamente isolado e grande parte de suas plantas aquáticas morre e se decompóe (Piedade, M.T.F., com. pess.).

Foram amostrados $21 \quad(\mathrm{n}=7)$ bancos de macrófitas aquáticas flutuantes, com predominância de E. crassipes, nos rios Solimóes (água branca), Negro (água preta) e na região de confluência destes rios (água mista). Para caracterizaçáo dos bancos foi estimada a riqueza e abundância de macrófitas aquáticas a eles associadas (Tabela 1 ).

Os parâmetros físicos da água, temperatura, oxigênio (YSI 55) e condutividade elétrica (YSI) foram medidos na superfície da água, no interior de cada um dos bancos de macrófitas amostrado. Outros parâmetros da água foram levantados da literatura (Tabela 2). Devido à ausência de separaçáo física entre os ambientes, a condutividade da água foi utilizada como critério para determinar o tipo da água. A condutividade foi escolhida também por estar ligada diretamente à concentração de nutrientes como $\mathrm{Na}, \mathrm{K}, \mathrm{Mg}$,

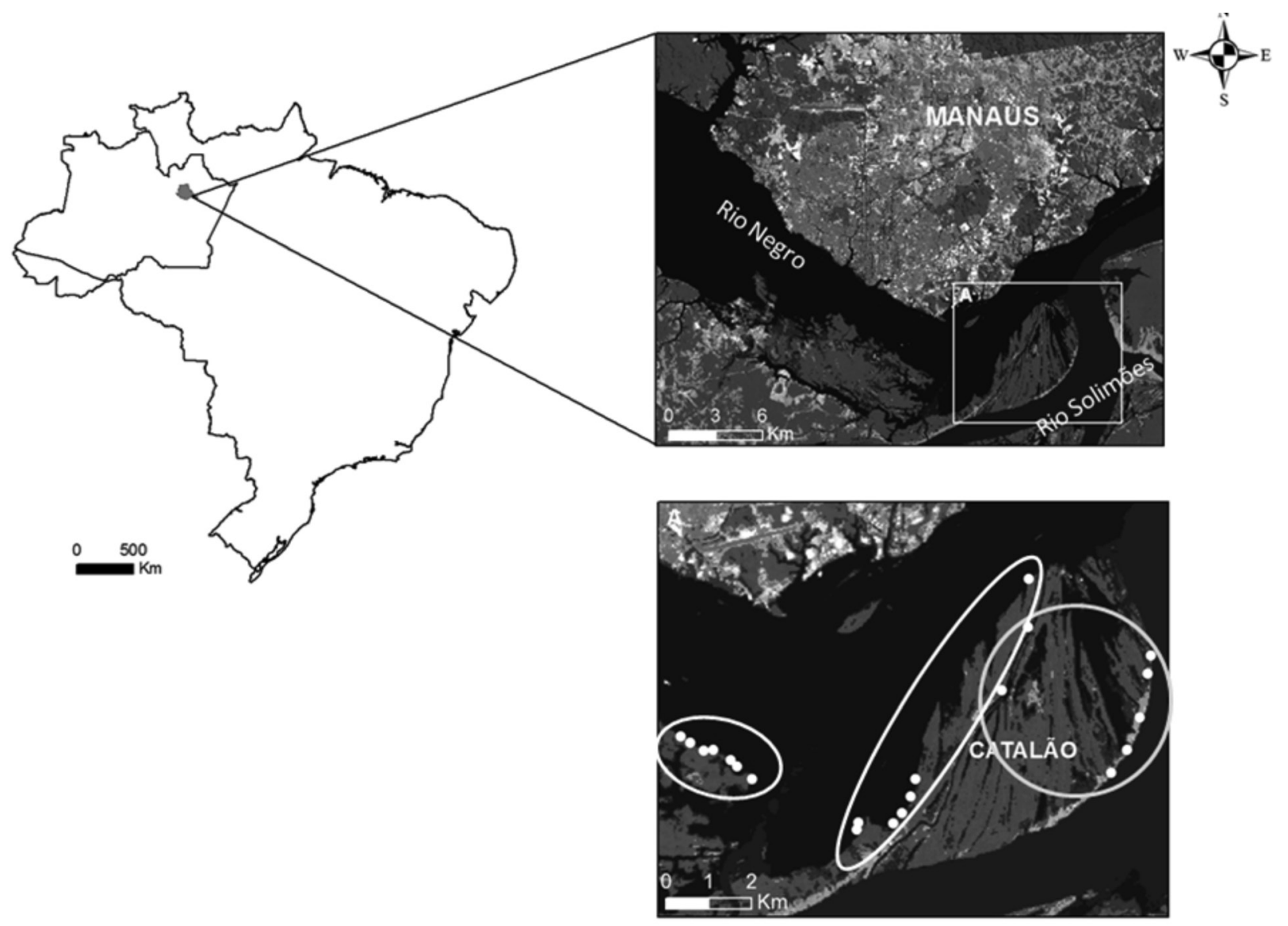

Figura 1 - Imagem Landsat da região do Catalão - Amazonas - Brasil, 2007. Círculo da esquerda destaca os pontos de água preta, 0 do meio os pontos de água mista e da direita os de água branca. (Elaborada por Juliana Peixoto). 
Ca (Junk e Furch 1980). Com base na condutividade da água e segundo a classificaçâo de Sioli (1984), as águas foram classificadas em: condutividade alta $\left(>50 \mu \mathrm{S} \mathrm{cm}^{-1}\right.$, água branca), média condutividade ( $>30 \mathrm{e} \leq 50 \mu \mathrm{sm}^{-1}$, água mista) e condutividade baixa ( $\leq 30 \mu \mathrm{S} \mathrm{cm}^{-1}$, água preta). Os pontos foram georreferenciados (GPS) mantendo-se uma distância mínima de $100 \mathrm{~m}$ para garantir a independência das amostras.

Os bancos de macrófitas foram escolhidos com base na dominância de E. crassipes. Em cada banco foram coletadas três amostras, distribuídas em diferentes locais do banco, utilizando para padronização um rapiché de $26 \mathrm{~cm}^{2}$ de borda, $48,2 \mathrm{~cm}$ de profundidade e malha de $1 \mathrm{~mm}$ (Esteves 1998). Todas as plantas coletadas possuíam uma altura mínima de $20 \mathrm{~cm}$. As raízes das macrófitas foram lavadas sobre peneira de $350 \mu \mathrm{m}$, para a remoção dos invertebrados aquáticos. Estes foram separados e fixados em álcool $70 \%$ para posterior identificação. A identificação foi realizada com o auxílio de lupa estereoscópica, até o nível de família de acordo com Pérez (1988) e Merritt e Cummnis (1996). Posteriormente, os organismos foram classificados conforme seus grupos tróficos funcionais (GTF) em coletores-catadores, coletores-

Tabela 1 - Macrófitas aquáticas associadas aos bancos de E. crassipes na região do Catalão, Amazonas, Brasil (junho de 2005).

\begin{tabular}{lccc}
\hline \multirow{2}{*}{ Macrófita aquática } & \multicolumn{3}{c}{ Tipos de água } \\
\cline { 2 - 4 } & Preta & Mista & Branca \\
\hline Paspalum repens & $\mathrm{X}$ & $\mathrm{X}$ & $\mathrm{X}$ \\
Pistia stratiotes & $\mathrm{X}$ & $\mathrm{X}$ & $\mathrm{X}$ \\
Salvinia auriculata & $\mathrm{X}$ & $\mathrm{X}$ & $\mathrm{X}$ \\
Pontederia rotundifolia & $\mathrm{X}$ & $\mathrm{X}$ & \\
Phyllantus fluitans & $\mathrm{X}$ & $\mathrm{X}$ & \\
Lemna sp. & & & $\mathrm{X}$ \\
Ludwigia natans & & $\mathrm{X}$ & \\
Ceratopteres pteridoides & & $\mathrm{X}$ & \\
\hline
\end{tabular}

Tabela 2 - Características bióticas e abióticas dos bancos de E. crassipes.

\begin{tabular}{lccc}
\hline \multirow{2}{*}{ Variáveis limnológicas } & \multicolumn{3}{c}{ Tipos de água } \\
\cline { 2 - 4 } & Preta & Mista & Branca \\
\hline Média da Condutividade $(\mu \mathrm{S} / \mathrm{cm})$ & 19,95 & 47,21 & 65,97 \\
\hline Média da Biomassa da Raiz $(\mathrm{g})$ & 3,67 & 5,21 & 5,64 \\
\hline $\begin{array}{l}\text { Média da Concentração de Oxigênio } \\
(\mathrm{mg} / \mathrm{L})\end{array}$ & 6,54 & 6,25 & 6,15 \\
\hline Média da Temperatura $\left({ }^{\circ} \mathrm{C}\right)$ & 29,31 & 29.23 & 28,48 \\
\hline Média do pH & $4,8-5,1^{*}$ & $6,4^{* *}$ & $6,7-6,9^{*}$ \\
\hline Material em suspensão $(\mathrm{mg} / \mathrm{mL})$ & - & $11,0^{* * *}$ & - \\
\hline $\mathrm{N}$ total $(\mu \mathrm{mol} / \mathrm{L})$ & $27,2^{*}$ & - & $43,1^{*}$ \\
\hline P total $(\mu \mathrm{S} / \mathrm{cm})$ & $0,22-0,81^{*}$ & - & $2-3,38^{*}$ \\
\hline
\end{tabular}

* Valores médios, nos rios Negro e Solimões entre 1969 e 1982 (Furch e Junk 1997). ** Valores médios nos lagos Poção e Padre (Catalão), (Alves e Darwich 2001).

*** Valor médio encontrado durante a cheia de 2005 no Catalão (Brito 2006). filtradores, fragmentadores, predadores e raspadores (Merritt e Cummins 1996); e depositados na Coleção Zoológica do INPA.

As raízes das macrófitas foram secas em estufa de ventilação forçada a $65^{\circ} \mathrm{C}$ até peso constante para obtenção do peso seco. Adicionalmente, foram coletados 40 indivíduos de E. crassipes nos quais foi medido o comprimento das raízes e depois estas foram secas em estufa para obtençấo do peso seco, conforme descrito acima, para estabelecer a relação entre biomassa e comprimento da raiz. Esta relaçáo foi testada por meio de correlação de Pearson.

A relaçáo entre a abundância, a riqueza de famílias e os grupos tróficos funcionais e as variáveis independentes (biomassa da raiz de E. crassipes e gradiente de condutividade) foi aferida por regressão linear simples. Para avaliar se a abundância, riqueza das famílias e dos grupos tróficos funcionais (GTF) diferiam entre os tipos de água foi utilizada análise de variância (ANOVA), com teste Tukey a posteriori para comparar as médias de cada categoria, e os valores $p$ corrigidos pelo método de Bonferroni.

Foi utilizada a técnica de ordenação indireta por meio do MDS (Escalonamento Multidimensional) para reduzir a composição da comunidade em duas dimensôes. O MDS não assume relaçốes lineares entre variáveis, não depende de um índice de associação específico e frequentemente resume mais informação em poucos vetores do que outros métodos de ordenaçáa (Manly 2004). A ordenação foi baseada em dados quantitativos, número de indivíduos por família e no número de grupos tróficos funcionais por família, utilizando a medida de distância (dissimilaridade) de Bray-Curtis. Para mensurar a qualidade do ajuste foi utilizada a medida stress, que indica a proporção da variaçáo das distâncias originais em relação às distâncias preditas pelo MDS. Os dois eixos resultantes das ordenaçóes das famílias e dos grupos tróficos foram utilizados como variáveis dependentes para realizaçáo de regressóes múltiplas a fim de verificar, separadamente, o efeito das variáveis condutividade e biomassa de raiz sobre a composição de macroinvertebrados. As análises estatísticas foram feitas no Programa Systat 10.2 e as ordenaçôes no Comunidata 1.5.

\section{RESULTADOS}

Foi encontrado um total de 1707 invertebrados aquáticos, distribuídos em 14 ordens, totalizando 35 famílias. Destas, 16 famílias estiveram presentes nos três tipos de água, três em dois tipos de água e $16 \mathrm{em}$ apenas um tipo de água. Os organismos mais abundantes foram Oligochaeta $(26,5 \%)$, Chironomidae $(23,8 \%)$ e Hydrophilidae $(15,75 \%)$. Dos grupos tróficos funcionais (GTF), os predadores foram os mais abundantes $(33,74 \%)$, seguidos pelos coletores-catadores 
e coletores-filtradores (ambos 28,12\%), raspadores $(6,1 \%)$ e fragmentadores $(3,93 \%)$.

A biomassa das raízes de E. crassipes apresentou uma correlação positiva com o comprimento destas $(r=0,69$, $\mathrm{p}<0,001$ ), náo variando entre os diferentes tipos de água, tendo sido observados valores médios de $1,06 \pm 0,4 \mathrm{~g}$ na água branca, $1,8 \pm 0,5 \mathrm{~g}$ na água mista e $1,2 \pm 0,8 \mathrm{~g}$ na água preta, portanto os tamanhos das amostras foram equiparáveis. A biomassa da raiz de E. crassipes foi positivamente relacionada à abundância e à riqueza de invertebrados aquáticos $\left(\mathrm{F}_{(1,19)}\right.$ $10,9, p=0,004, \mathrm{r}^{2}=0,4 ; \mathrm{F}_{(1,19)}=6,8, p=0,017, \mathrm{r}^{2}=0,3$ respectivamente) (Figura $2 \mathrm{~A} \mathrm{e} \mathrm{B}$ ).

A abundância de invertebrados aquáticos não apresentou relação com o gradiente de condutividade $(p=0,89)$, porém variou significativamente com o tipo de água $\left(\mathrm{F}_{(2,18)}=13,3\right.$, $p<0,001, \mathrm{r}^{2}=0,6$ ) (Figura 2C). Os indivíduos de E. crassipes que foram coletados na água mista apresentaram maior abundância de invertebrados aquáticos, do que em água branca $(p=0,002)$ e em água preta $(p=0,003)$. A riqueza de invertebrados aquáticos não apresentou relaçáo com o gradiente de condutividade $(p=0,425)$, embora tenha variado significativamente $\left(\mathrm{F}_{(2,18)}=6,1, p=0,009, \mathrm{r}^{2}=0,4\right)$ com o tipo de água (Figura 2D). Entretanto, a diferença de riqueza só foi estatisticamente maior na água mista em comparação com a água preta (Tukey; $p=0,02$ ).

Quando a comunidade foi separada em GTF as abundâncias dos coletores-catadores, coletores-filtradores e raspadores variaram entre locais com tipos de água diferentes $\left(\mathrm{F}_{(2,18)}=11,03, p=0,001, \mathrm{r}^{2}=0,55 ; \mathrm{F}_{(2,18)}=6,52, p=0,007\right.$, $\mathrm{r}^{2}=0,42 ; \mathrm{F}_{(2,18)}=8,38, p=0,0039, \mathrm{r}^{2}=0,48$, respectivamente; Figura 3). Para os coletores-catadores (Figura $3 \mathrm{~A}$ ) e raspadores
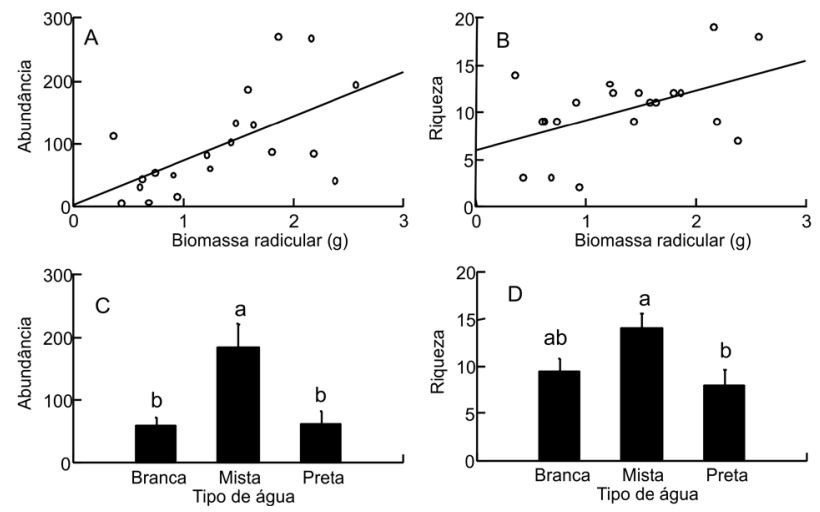

Figura 2 - (A) Relação entre a abundância de invertebrados aquáticos e a biomassa da raiz de E. crassipes; (B) Relação entre a riqueza de invertebrados aquáticos e a biomassa da raiz de E.crassipes; (C) Abundância de invertebrados aquáticos pelo tipo de água e (D) riqueza de invertebrados aquáticos segundo o tipo de água na região do Catalão, Amazonas/Brasil, em junho de 2005. Valores médios com erros padrões $(n=7)$. Médias com letras iguais não são significativamente diferentes.
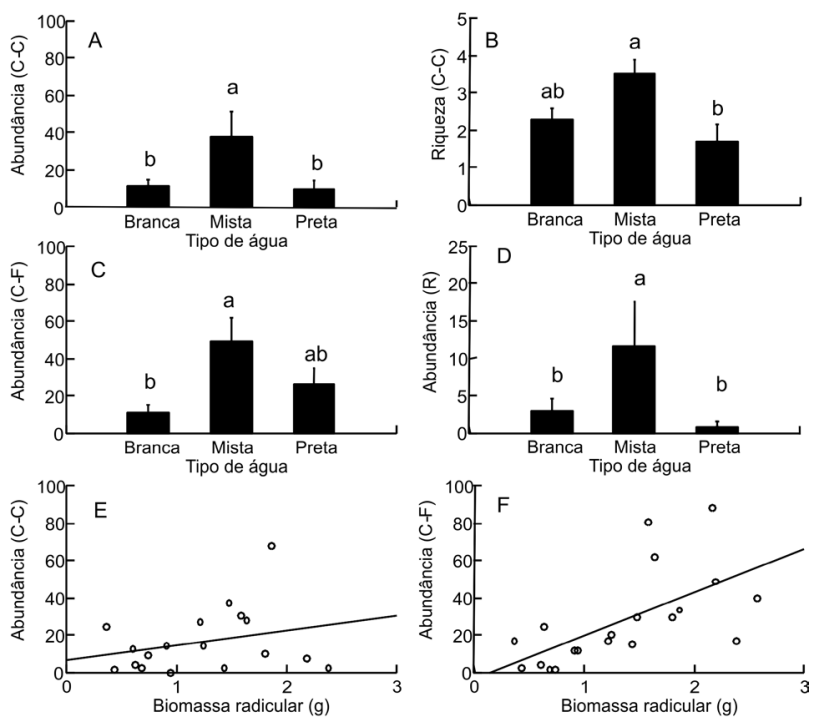

Figura 3 - Invertebrados aquáticos conforme seus grupos tróficos funcionais ocorrendo em raízes de E. crassipes, na região do Catalão, Amazonas/Brasil, em junho de 2005. A,C,D,E,F) abundância; B) riqueza. Onde: $C-C=$ coletorescatadores $; \mathrm{C}-\mathrm{F}=$ coletores-filtradores $; \mathrm{R}=$ Raspadores. Valores médios com erros padrões $(n=7)$. Médias com letras iguais não são significativamente diferentes.

(Figura 3D) a abundância foi maior na água mista que nos outros tipos de água, enquanto que para os coletores-filtradores (Figura 3C) a abundância foi maior somente na água mista em relação à água preta (Figura 3B). Somente a riqueza dos raspadores (Figura 3D) variou significativamente com o tipo de água $\left(\mathrm{F}_{(2,18)}=4,75, p=0,022, \mathrm{r}^{2}=0,34\right)$. Neste caso, a água mista possuiu uma riqueza maior de raspadores que a água preta (Tukey; $p=0,018$ ). Os demais grupos tróficos não apresentaram diferença de riqueza significativa com o tipo de água (ANOVA, $p<0,05$ ). Não houve relação entre a riqueza de nenhum dos grupos tróficos com a condutividade elétrica da água (Regressão, $p>0,05$ ).

A abundância dos coletores-catadores e coletoresfiltradores foi positivamente relacionada com a biomassa das raízes de $E$. crassipes $\left(\mathrm{F}_{(1,19)}=29,15, p<0,001, \mathrm{r}^{2}=0,6 ; \mathrm{F}_{(1,}\right.$ 19) $=20,95, p<0,001, \mathrm{r}^{2}=0,52$; respectivamente; Figura 3 E, F). Porém, a riqueza de nenhum dos grupos tróficos foi relacionada à biomassa de raízes (regressão, $p>0,05$ ).

A análise de escalonamento multidimensional resultou em uma ordenação das espécies e sítios em duas dimensóes, com stress de 0,01 e proporção da variância (RSQ) de 0,736. A ordenação produzida para os sítios conseguiu distinguir 2 grupos com base na categorização utilizada para água (Figura 4), separando a água mista e agrupando a preta e branca. A maioria das famílias ocorreu em todo o gradiente, não havendo, assim, estruturação da abundância de invertebrados aquáticos segundo o gradiente de condutividade (Figura 5b) 
(Pillai Trace $=0,07, \mathrm{~F}_{(2,18)}=0,65, p=0,53$; Figura 5A). Algumas famílias como Gomphidae, Hidracarina e Leptophlebiidae ocorreram somente em locais com raízes menos densas; outras como Hydroptilidae, Leptoceridae e Megapodogrionidae, ocorreram somente associadas a plantas com grande biomassa radicular. Assim, houve uma estruturação da abundância dos

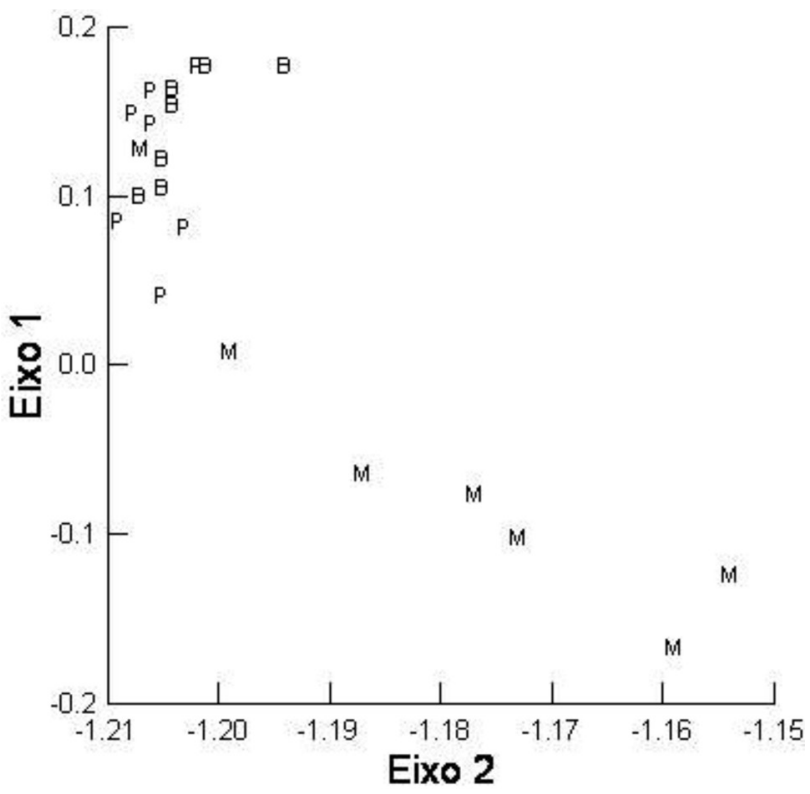

Figura 4 - Ordenação resultante da análise de escalonamento multidimensional (MDS) dos sítios. Onde: $\mathrm{B}=$ água branca; $\mathrm{M}=$ água mista; $\mathrm{P}=$ água preta.

macroinvertebrados em funçáo da biomassa de raiz de $E$. crassipes $\left(\right.$ Pillai Trace $=0,38, \mathrm{~F}_{(2,17)}=5,46, p=0,01$; Figura 5B).

\section{DISCUSSÃO}

No rio Negro, poucas são as espécies de macrófitas aquáticas flutuantes encontradas (Junk e Mello 1990; Piedade et al. 2010b). Contudo, uma maior abundância destas plantas na área de estudo pode ser influenciada pela conectividade entre os rios Negro e Solimóes que ocorre sazonalmente (Leite et al. 2006). A confluência desses dois rios leva a um aporte de nutrientes oriundos da água branca para o lago Cataláo, durante a fase aquática (Leite et al. 2006). Assim, as macrófitas flutuantes e os propágulos carregados pela correnteza conseguem sobreviver por um longo período de tempo em águas com baixa qualidade nutricional devido à sua grande capacidade de estocagem de nutrientes (Esteves 1982).

Nesse estudo, a abundância e riqueza total de invertebrados aquáticos no lago Catalão foram maiores na água com média condutividade (mista) que nos outros tipos de água, não havendo diferença entre a abundância e a riqueza de espécies entre a água preta (baixa condutividade) e branca (alta condutividade), mostrando que, apesar da melhor qualidade nutricional na água branca (Furch e Junk 1997), as águas mistas têm maior abundância e riqueza de espécies. Regiôes de ecótono costumam possuir maior número de organismos por apresentar mais micro-hábitats que áreas adjacentes (Gosz 1993; Risser 1993). A relação positiva encontrada entre a abundância e a riqueza de famílias e a biomassa da raiz de $E$. crassipes, considerando que náo houve relaçáo entre o peso da raiz e o tipo de água, indica que o tipo de água não é o principal fator responsável por estes atributos e sim a estrutura do hábitat.

A forma de exploração dos diversos recursos alimentares pelos invertebrados pode ser obrigatória ou facultativa, dependendo das adaptaçóes morfológicas e comportamentais de cada grupo (Cummins e Klug 1979; Mihuc 1997). As formas especialistas obrigatórias, com dieta muito restrita, deslocam-se para outras áreas mais rapidamente do que as generalistas facultativas, cuja fonte alimentar é variável (vegetal e/ou animal) (Cummins e Klug 1979). Estas últimas são mais tolerantes sob condiçôes de distúrbio, pois conseguem se adaptar mais facilmente a mudanças no tipo e na disponibilidade de alimento (Cummins e Klug 1979). No presente estudo, os coletores-catadores, coletores-filtradores e raspadores foram mais abundantes na água mista e, os dois primeiros, em raízes mais pesadas. Porém, somente a riqueza dos raspadores variou em função do tipo de água. A maior riqueza e abundância de raspadores em plantas que possuíam raízes maiores era esperada, por estas apresentarem uma superfície de contato maior, que, consequentemente, propiciaria a formaçáo de biofilme mais eficiente, permitindo uma maior disponibilidade de alimento para esses organismos (Vannote et al. 1980).

A presença e abundância dos vários grupos tróficos funcionais e a dominância de representantes obrigatórios ou facultativos sấo reflexo direto da disponibilidade dos recursos alimentares necessários (tanto em quantidade como em qualidade) e dos parâmetros ambientais relacionados (Merritt e Cummins 1996). A maior abundância dos coletores-catadores e coletores-filtradores indica maior quantidade de matéria orgânica no ambiente (Marques et al. 1999). Por isso, seria esperado observar maior número de espécies de coletorescatadores e coletores-filtradores em águas brancas, onde foi observada maior condutividade e maior concentração de nutrientes (Junk e Furch 1980). Entretanto, embora no rio Solimôes haja uma elevada quantidade de material alimentar de alto valor energético em suspensão, este recurso está associado a águas bastante túrbidas devido a uma enorme carga sedimentar, notadamente em períodos de grande fluxo de corrente. Esta condiçấo pode dificultar a obtençấo de alimento, pois a alta carga sedimentar não é favorável para esses organismos porque suas estruturas de captação e filtragem não são adaptadas a essas condiçóes extremas (Hardy 1989; 


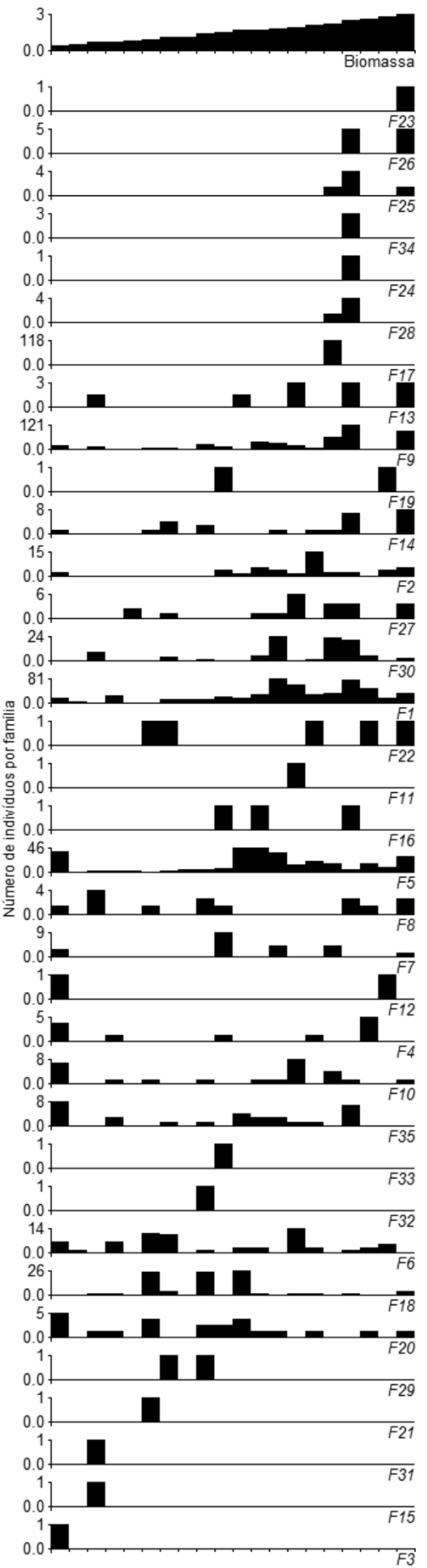

Biomassa radicular $(g)$

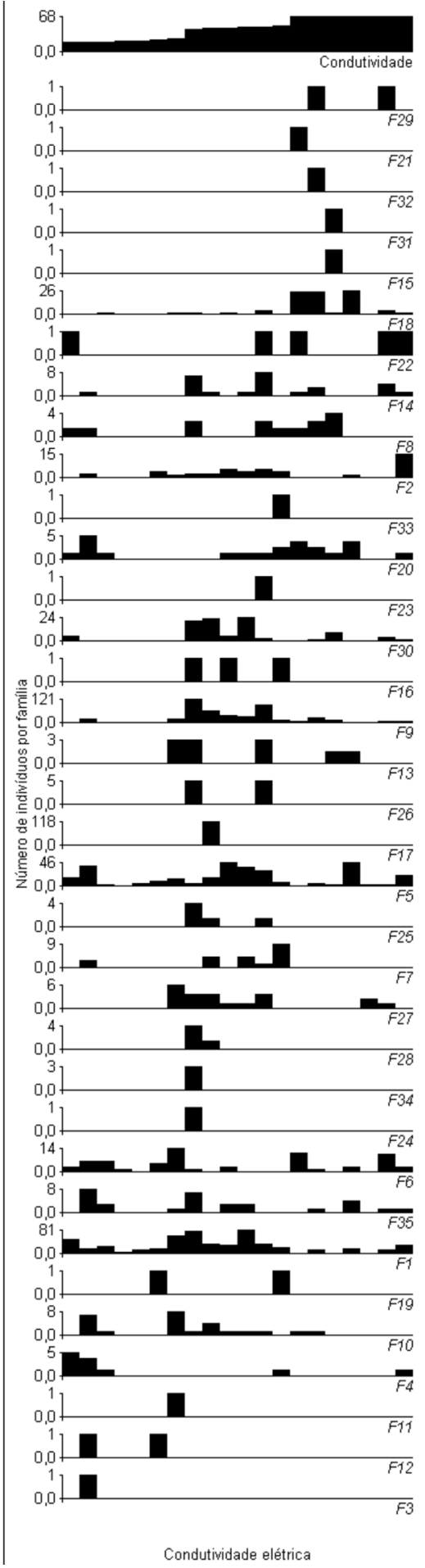

Condutividade elétrica

Figura 5 - Ordenação das famílias de macroinvertebrados aquáticos segundo: a) gradiente de biomassa radicular (g) de E. crassipes; e b) gradiente de condutividade elétrica $(\mu \mathrm{S} / \mathrm{cm})$ na região de confluência dos rios Negro e Solimões, junho de 2005. Os números indicam as famílias: 1 Oligochaeta; 2 Hirudinea ; 3 Hidracarina; 4 Elmidae; 5 Hydrophilidae; 6 Noteridae; 7 Scirtidae; 8 Ceratopogonidae; 9 Chironomidae; 10 Dytiscidae; 11 Empididae; 12 Tabanidae; 13 Baetidae; 14 Leptohyphidae; 15 Leptophlebiidae; 16 Polymitarcyidae; 17 Belostomatidae; 18 Corixidae; 19 Naucoridae; 20 Lepidoptera; 21 Gomphidae; 22 Libellulidae ; 23 Megapodagrionidae; 24 Coenagrionidae; 25 Hydroptilidae; 26 Leptoceridae ; 27 Philopotamidae; 28 Polycentropodidae; 29 Ancylidae; 30 Biomphalaria; 31 Physidae; 32 Lymnaeidae; 33 Thiaridae; 34 Bivalvia (classificado até classe); 35 Paleomonidae. 
Stewart e Downing 2008). Desta forma, os organismos podem viver melhor em ambientes que, embora possuam menor quantidade de matéria orgânica alimentar disponível, ainda são relativamente ricos em nutrientes, e estes podem ser mais facilmente incorporados devido à carga sedimentar mais baixa, como verificado nas águas mistas.

A ocorrência de predadores foi homogênea em todo o gradiente de condutividade e de biomassa das raízes. Essa distribuição de predadores em todo gradiente é esperada, pois eles dependem diretamente da ocorrência de outros invertebrados e não da disponibilidade de partículas orgânicas (Vannote et al. 1980; Marques et al. 1999). A maior abundância de fragmentadores na água mista pode estar relacionada à maior disponibilidade de detritos (Hardy 1989), a qual não foi quantificada.

Não foi observada relação entre a composição da comunidade dos invertebrados aquáticos e o gradiente de condutividade, sugerindo que, durante o período estudado, a concentração de nutrientes não foi o fator primordial para a distribuiçấo das famílias de invertebrados aquáticos. Por outro lado, foi observada a distribuição das famílias em função do gradiente de biomassa de raízes, mostrando que a estrutura do hábitat é um fator importante para o estabelecimento dos organismos. Alguns autores sugerem que a complexidade espacial inata da arquitetura de uma planta individualmente cria uma diversidade de hábitats importante para macroinvertebrados e peixes (Lille e Budd 1992; Dibble et al. 1996; Taniguchi et al. 2003; Dibble e Thomaz 2006). Para os invertebrados aquáticos associados a E. crassipes, a estruturação da comunidade, ou seja, a distribuição das famílias ao longo do gradiente, foi explicada pela variação na biomassa da raiz que, quanto maior, propiciou uma maior complexidade de hábitat.

\section{CONCLUSÕES}

As raízes mais densas de E. crassipes mostraram-se microhábitats favoráveis para os organismos, oferecendo abrigo, local favorável para captura de presas pelos predadores, e para captaçấo de alimento pelos coletores-catadores, coletoresfiltradores e raspadores que utilizam direta ou indiretamente o biofilme formado sobre as raízes. Desta forma, a organização da comunidade de macroinvertebrados aquáticos está relacionada ao gradiente de biomassa de raízes, mostrando que a estrutura do hábitat é um fator importante para o estabelecimento desse grupo de organismos.

\section{AGRADECIMENTOS}

Agradecemos aos Drs. Bruce Forsberg, Jansen Zuanon e Rosseval Leite pelos comentários ao projeto; aos revisores anônimos pelas valiosas sugestôes; ao CNPq; e à Fundação de Amparo à Pesquisa do Amazonas (FAPEAM) pelas bolsas de mestrado concedidas.

\section{BIBLIOGRAFIA CITADA}

Adis, J.; Junk, W.J. 2002. Terrestrial invertebrates inhabiting lowland river floodplains of Central Amazonia and Central Europe: a review. Freshwater Biology, 47: 711-731.

Ali, M.M.; Mageed, A.A.; Heikal, M. 2007. Importance of aquatic macrophyte for invertebrate diversity in large subtropical reservoir. Limnologica, 37: 155-169.

Amoros, C.; Bornette, G. 2002. Connectivity and biocomplexity in waterbodies of riverine floodplains. Freshwater Biology, 47: 761-776.

Bohonak, A.J.; Jenkins, D.G. 2003. Ecological and evolutionary significance of dispersal by freshwater invertebrates. Ecology Letters, 6: 783-796

Callisto, M., Esteves, F. de A., Gonçalves, J.F. Jr.; Fonseca, J.J.L. 1998. Benthic macro-invertebrates as indicators of ecological fragility of small rivers ('igarapés') in a bauxite mining region of Brazilian Amazonia. Amazoniana, 15(1/2): 1-9.

Callisto, M.; Moretti, M.; Goulart, M.D.C. 2001. Benthic macroinvertebrates as a tool of river health assessment. Revista Brasileira de Recursos Hidricos, 6 (1): 71-82. (in Portuguese)

Cheruvelil, K.S.; Soranno, P.A.; Serbin, R.D. 2000. Macroinvertebrates associated with submerged macrophytes: sample size and power to detect effects. Hydrobiologia, 441: 133-139.

Cronin, G.; Lewis Jr., W.M.; Schiehser, M.A. 2006. Influence of freshwater macrophytes on the littoral ecosystem structure and function of a young Colorado reservoir. Aquatic Botany, 85: $37-43$.

Cummins, K.W.; Klug, M.J., 1979. Feeding ecology of stream invertebrates. Annual Review of Ecology and Systematcs, 10: 145-172.

Dibble, E.D.; Thomaz, S.M. 2006. A Simple Method to Estimate Spatial Complexity in Aquatic Plants. Brazilian Archives of Biology and Technology, 49(3): 421-428.

Dibble, E.D.; Killgore, K. J.; Dick, G. O. 1996. Measurement of plant architecture in seven aquatic plants. Journal of Freshwater Ecolology, 11: 311-318.

Esteves, F.A. 1982. Biomass and analysis of the major inorganic components of floating aquatic macrophytes Eichhornia crassipes (Mart.) Solms in six reservoirs of São Paulo State, Brazil. Ciência e Cultura, 34: 1197-1200.

Esteves, F.A. 1998. Fundamentals of Limnology Rio de Janeiro - RJ. Ed. Interciencia / FINEP. 575 pp. (in Portuguese)

Franklin, E.; Schubart, H.O.R.; Adis, J. 1997. Edaphic mites of two flooded Forests of Central Amazon: vertical distribution, abundance and soil recolonization after flood. Brazilian Journal of Biology, 57 (3): 501-520. (in Portuguese)

Furch, K.; Junk, W. J. 1997. Physico-chemical conditions in floodplains lakes, p. 69-108. In: W.J. Junk. (Org.). The Central Amazon Floodplain: Ecology of a Pulsing System. 1 ed. Berlin: Springer Verlag. $126 \mathrm{pp}$. 
Gosz, J.R. 1993. Ecotone hierarchies. Ecological Applications, 3 (3): 369-376.

Hardy, E.R. 1989. Effects of temperature, food concentration and turbidity on life cycle characteristics of planktonic cladocerans in a tropical lake, Central Amazon: field and experimental work. Thesis (PhD), Royal and Bedford New College, London. 337 pp.

Holland, M.M. 1988. SCOPE/MAB Technical consultations on landscape boundaries. Report of a SCOPE/MAB workshop on ecotones, p. 47-106. In: di Castri, F.: Hansen, A.J.; Holland, M.M. (Eds). A new look at ecotones: emerging international projects on landscape boundaries. Biology International, Special Issue 17.

Junk, W.J.; Furch, K. 1980. Water Chemistry and aquatic macrophytes of rivers and streams in Amazon river Basin and adjacent areas. Part I: Cuiabá - Porto Velho - Manaus Path. Acta Amazonica, 10 (3): 611-633. (in Portuguese)

Junk, W.J.; Howard-Williams, C. 1984. Ecology of aquatic macrophytes in Amazonia, p. 269-293. In: Sioli, H. (Ed). The Amazon, Limnology and landscape ecology of a mighty tropical river and its basin. Monographiar Biologicae. Dr. W. Junk Publ., Dordrecht.

Junk, W.J.; Piedade, M.T.F. 1993. Herbaceous plants of the Amazon floodplain near Manaus: Species diversity and adaptations to the flood pulse. Amazoniana, 12(3/4): 467-484.

Junk, W.J. 1973. Investigations on the ecology and productionbiology of the "floating meadows" (Paspalo-Echinochloetum) on the middle Amazon. Part II. The aquatic fauna in the root zone of floating vegetation. Amazoniana, 4(1): 9-102.

Junk, W.J. 1983. As águas da regiāo Amazônica. In: Amazônia; desenvolvimento, integração, ecologia. Brasiliense, São Paulo.

Junk, W.J., Bayley, P.B.; Sparks, R.E. 1989. The flood pulse concept in river - floodplain systems, p. 110-127. In: Dodge, D.P. (Ed). Proceedings of the International Large River Symposium. Publ. Canadian Journal of Fisheries and Aquatic Sciences, 106.

Junk, W.J.; Mello, J.A.S.N. 1990. Ecological impacts of hydroelectric dams in Brazilian Amazon Basin. Estudos Avançados, 4(8):126143. (in Portuguese)

Junk, W.J.; Piedade, M.T. 1997. Plant life in the floodplain with special reference to herbaceous plants, p. 147-181.in: Junk, W.J. (Ed.) The Central Amazon Floodplain. Springer-Verlag, New York 126.

Larcher, W. Physiological plant ecology. Plants under stress. Springer. Austria. 1995.513 pp.

Leite, R.G.; Silva, J.V.V.; Freitas, C.E. 2006. Abundance and distribution of fish larvae in Cataláo Lake and in the confluence of the Solimóes and Negro River, Amazon, Brazil. Acta Amazonica, 36 (4): 557 - 562. (in Portuguese)

Lillie, R.A.; Budd, J. 1992. Habitat architecture of Myriophyllum L. as an index to habitat quality for fish and macroinvertebrates. Journal of Freshwater Ecolology, 7:113-125.

Manly, B.J. Multivariate statistical methods: a primer. 3 ed. Chapman and Hall. New York. 2004. 214 pp.
Marques, M.G.S.M.; Ferreira, R.L.; Barbosa, F.A.R. 1999. Aquatic macroinvertebrate community and limnological characteristics of 'Carioca' and 'da Barra' lakes, Parque Estadual do Rio Doce, MG. Revista Brasileira de Biologia, 59 (2): 203-210. (in Portuguese).

Martius, C. 1987. The adaptation of termites (Nasutitermes sp. Termitidae, Nasutitermitinae) to Amazonian inundation forests, p. 609-610. In: Eder, J.; Rembold, H. (eds.). Chemistry and biology of social insects. Peperny, München.

Merritt, R.W.; Cummins, K.W. 1996. An introduction to the aquatic insects of North America. 3rd edition. Kendall/Hunt Publishing Company, Iowa. USA. 862 pp.

Mihuc, T.B. 1997. The functional trophic role of lotic primary consumers: generalist versus specialist strategies. Freshwater Biology, 37: 455-462.

Nessimian, J.L., Dorvillé, L.F.M., Sanseverino, A.M.; Baptista, D.F. 1998: Relation between flood pulse and functional composition of the macroinvertebrate benthic fauna in the lower Rio Negro, Amazonas, Brazil. Amazoniana, 15(1/2): 35-50.

Pérez, G.R. 1988. Guia para el estudio de los macroinvertebrados acuáticos del Departamento de Antioquia. Fondo Fen. Colombia/ Colciencias, Universidade de Antioquia. 132 pp.

Petruccio, M.M.; Esteves, F. A. 2000. Uptake rates of nitrogen and phosphorus in the water by Eichhornia crassipes and Salvinia auriculata. Revista Brasileira de Biologia, 60(2): 229-236.

Piedade, M.T.F.; Ferreira, C.S.; Franco, A. 2010a. Reproductive strategies of vegetation and their responses to the flood pulse in floodplains of the Central Amazon. Revista Ecosistemas, 19: 1-4 (online) (in Spanish)

Piedade, M.T.F.; Junk, W.J.; D’Angelo, S.A.; Wittmann, F; Schoengart, J; Lopes, A. 2010b. Aquatic herbaceous plants of the Amazon floodplains: state of the art and research needed. Acta Limnologica Brasiliensia, 2: 165-178.

Resh, V.H.; Myers, M. e Hannaford, M.J. 1996. Macroinvertebrates as Biotic Indicators of Environmental Quality, p. 647-667. In: Hauer, F.R.; Lamberti, G.A. (Eds.). Methods in Stream Ecology. Academic Press, San Diego.

Risser, P.G. 1993. Ecotones at Local to Regional Scales from Around the World. Ecological Applications, 3 (3): 367-368.

Sculthorpe, C. D. 1985. The Biology of Aquatic Vascular Plants. Koeltz Scientific Books, Königstein/West Germany. 610 pp.

Sioli, H. 1968. Hydrochemistry and geology in the Brazilian Amazon region. Amazoniana, 1 (3): 267-277.

Sioli, H. 1984. The Amazon and its main affluents: Hydrography, morphology of the river courses, and river types. p. 127-165. In: Sioli, H. (Ed.) The Amazon. Limnology and landscape ecology of a mighty tropical river and its basin. Monographiar Biologicae, 56. Dr. W. Junk Publ., Dordrecht.

Sousa, A. K. F. 2008. Seasonal variation and spatial concentration of the trace elements in rivers Urucu, Solimöes and Negro - Western Amazon, Brazil. Dissertação de Mestrado, Universidade Federal do Amazonas, Manaus. 229 pp. (in Portuguese) 
Stewart, T.W.; Downing, J.A. 2008. Macroinvertebrate communities and environmental conditions in recently constructed wetlands. Wetlands, 28 (1):141-150.

Taiz, L.; Zeiger, E. 2004. Plant Physiology. Third ed. Atmed, Porto Alegre. 719 pp. (in Portuguese)

Taniguchi, H.; Nakano, S.; Tokeshi, M. 2003. Influences of habitat complexity on the diversity and abundance of epiphytic invertebrates on plants. Freshwater Biology, 48: 718-728.
Van den Berg, M.S.; Coops, H.; Noordhuis, R.; Van Schie, J.; Simons, J. 1997. Macroinvertebrate communities in relation to submerged vegetation in two Chara-dominated lakes. Hydrobiologia, 342/343: 143-150.

Vannote, R.L.; Minshal, G.W.; Cummins, K.W.; Sedell, J.R.; Cushing, C.E. 1980. The River Continuum Concept. Canandian Journal of Fisheries and Aquatic Sciences, 37: 130-137.

Recebido em 28/06/2010

Aceito em 14/11/2010 